\title{
Relative Reflection Difference as a Method for Measuring the Thickness of the Exfoliated $\mathrm{MoSe}_{2}$ Layers
}

\author{
K. Łempicka*, K. Norowski, M. Grzeszczyk, M. Król, K. Lekenta, A. Babiński, \\ B. PiętKa And J. SzCZYTKO
}

Institute of Experimental Physics, Faculty of Physics, University of Warsaw, Pasteura 5, 02-093 Warsaw, Poland

\begin{abstract}
We propose a method for measuring the thickness of the exfoliated $\mathrm{MoSe}_{2}$ layers deposited on $\mathrm{Si} / \mathrm{SiO}_{2}$ substrate, based on the reflectance measurements performed with laser light illumination at two different wavelengths: red and green from confocal microscope at room temperature. We demonstrate the correlation between the number of layers in a flake and the value of its relative reflection difference. We applied the transfer matrix method to calculate the reflectivity and verify our experimental results. The approach proposed by us allows for fast and automatic verification of the exfoliated $\mathrm{MoSe}_{2}$ layers thickness on large areas of the substrate.
\end{abstract}

DOI: 10.12693/APhysPolA.132.316

PACS/topics: 63.20.dd, 78.20.Ci, 78.30.-j, 78.66.Li

\section{Introduction}

Transition-metal dichalcogenide crystals (TMDCs) have emerged as a new class of materials that show exceptional physical and optical properties [1]. As opposed to graphene, which does not have a band gap, the monolayer of these materials has a direct bandgap, thus makes them promising candidates for applications as light emitters, detectors, digital electronics and photovoltaic devices [2-4].

One of the typical representative compound of the TMDCs semiconducting family is molybdenum diselenide $\left(\mathrm{MoSe}_{2}\right)$. Structure of this synthetic crystal is characterized by strong intralayer covalent bonds within planes of hexagonally arranged selenium and molybdenum atoms and weakly interacting Se-Mo-Se layers [5]. To obtain thin layers of $\mathrm{MoSe}_{2}$ one can use exfoliation technique based on adhesive and elastomeric tapes [6]. However, this process is difficult to control and typically flakes of different thickness are deposited on the substrate. The optical microscope can be used for previsualisation and evaluation of the quality of the samples, but the exact number of monolayers in each flake can be hardly estimated.

An easy, quick and nondestructive method of the determination of layer thickness on large area substrates is important for commercial applications and research. In order to effectively determine the number of layers of $\mathrm{MoSe}_{2}$ and visualize macroscopic defects we propose reflectance measurements performed at two different wavelengths $(543 \mathrm{~nm}$ and $650 \mathrm{~nm})$. The analysis of the difference of relative (i.e. normalized to the substrate) reflectance from confocal microscope can be used to determine the exact number of TMDC layers. Our experi-

\footnotetext{
* corresponding author; e-mail k. lempicka@student.uw.edu.pl
}

mental results are accompanied with theoretical simulations based on transfer matrix method, which shows good agreement with the experiment. We compare and validate our approach with the Raman scattering measurements, standard method in determination of the thickness of TMDCs thin layers.

\section{Experimental setups}

The thin $\mathrm{MoSe}_{2}$ layers were prepared by the polydimethylosiloxan-based exfoliation technique from bulk crystal of $\mathrm{MoSe}_{2}$ purchased from HQ Graphene. Exfoliated thin flakes were deposited onto a silicon substrate covered by $75 \mathrm{~nm}$ thick silica oxide top layer.

The samples were studied using standard optical microscopy technique. The reflection measurements were carried out using green $(543 \mathrm{~nm})$ and red $(650 \mathrm{~nm})$ laser light illumination in the confocal microscopy Attocube CFM II system. In the Raman scattering measurements we used a green $532.8 \mathrm{~nm}(2.33 \mathrm{eV})$ laser with $250 \mu \mathrm{W}$ power at the sample. The $100 \times$ microscope objective was used and the diameter of the laser spot was $1 \mu \mathrm{m}$. The spectrometer grating of 2400 grooves/mm allows for a spectral resolution of $1.3 \mathrm{~cm}^{-1}$. All measurements were performed at ambient temperature.

\section{Results}

The optical microscopy was used for previsualization and evaluation of the quality of the samples. In order to improve optical contrast and spatial resolution a confocal microscope was used for scanning the surface of TMDCs samples [7] and for studying different macroscopic defects formed during the exfoliation process. The images of relative reflectance difference (RRD) were obtained using the formula:

$$
\mathrm{RRD}=\frac{I\left(\mathrm{MoSe}_{2}, 543\right)}{I\left(\mathrm{SiO}_{2}, 543\right)}-\frac{I\left(\mathrm{MoSe}_{2}, 650\right)}{I\left(\mathrm{SiO}_{2}, 650\right)},
$$

where $I(X, \lambda)$ is the intensity of the green $(\lambda=543 \mathrm{~nm})$ 
or red $(\lambda=650 \mathrm{~nm})$ light reflected from the flake $\left(X=\mathrm{MoSe}_{2}\right.$ or $\left.\mathrm{SiO}_{2}\right)$. The dark signal of the detector (i.e. without illumination) was taken into account.
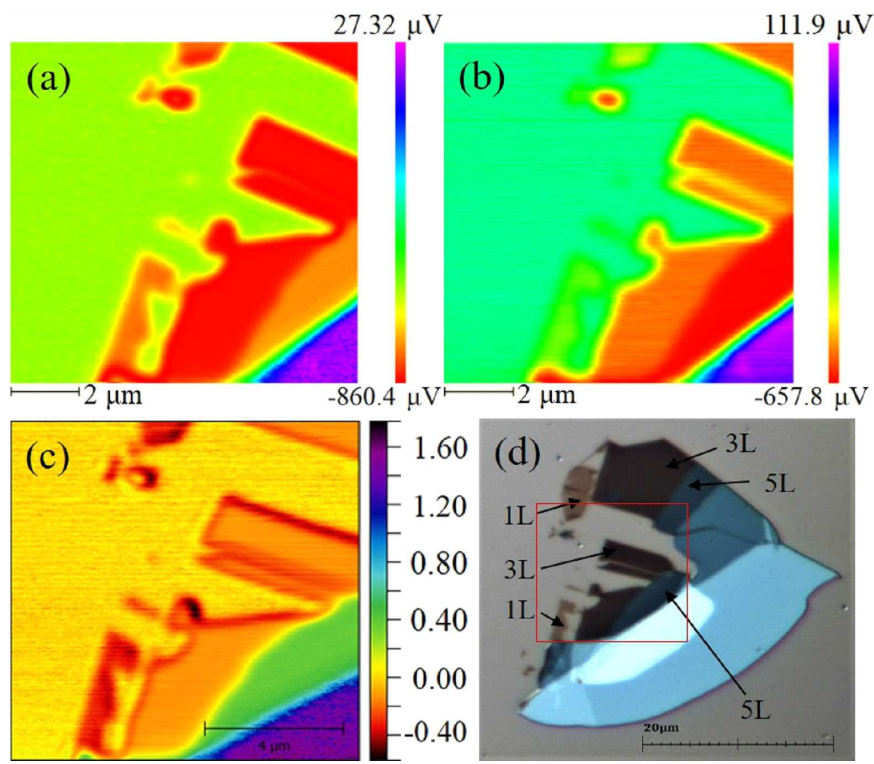

Fig. 1. The images of the reflectance from the TMDCs sample form confocal microscope with (a) green $(543 \mathrm{~nm})$ and (b) red laser $(650 \mathrm{~nm})$ illumination, (c) calculated RRD map, and (d) optical microscope image with white illumination. The red square marked in Fig. 1d shows the scanning area of confocal microscope. The arrows indicate the places measured by Raman spectroscopy. Labels " $1 \mathrm{~L}$ " " $5 \mathrm{~L}$ " indicate the number of layers.

In Fig. 1 the procedure of calculating RRD is presented. First, reflectivity intensity map for green and red illumination were collected as is presented in Fig. 1a and $\mathrm{b}$. The investigated area contains flakes of different thickness. Depending on the number of monolayers the monochromatic illuminations with different wavelengths distinguishes different parts of the sample due to interferences between light reflected from each interface [8]. Due to the small overall contrast at both of the investigated wavelengths, Eq. (1) was used to calculate the RRD map which is shown in Fig. 1c. This procedure significantly improved contrast of the image, and clearly distinguished flakes with different thickness.

The same area of the sample was investigated by the Raman spectroscopy (Fig. 1d). The Raman spectra of flakes of various thickness are presented in Fig. 2. Characteristic silica Raman $520.7 \mathrm{~cm}^{-1}$ signal from the substrate was used for precise calibration of energies of $\mathrm{MoSe}_{2}$ Raman spectra. The thicknesses of the layers were obtained by analyzing energy, width, intensity and shape of the peaks in the Raman scattering [9]. Especially, three vibrational modes play a key role in this analysis: $E_{2 g}^{1}$ (active out-of plane vibrational mode), $A_{1 g}$ (active in-plane vibrational mode) and $B_{2 g}^{1}$ (inactive out-of plane vibrational mode). These modes are strongly influenced

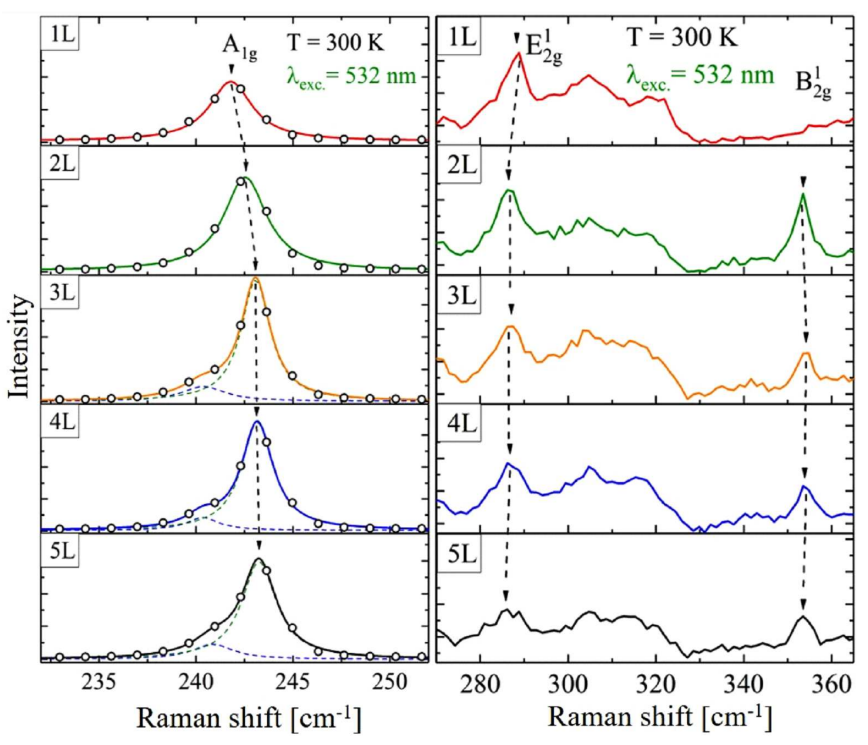

Fig. 2. Raman spectra of a few-layer $\mathrm{MoSe}_{2}$. Labels "1L" " $5 \mathrm{~L} "$ indicate the number of layers.

by the thickness of the flakes. The out-of-plane mode $A_{1 g}$ was observed in spectral range of $241.5-243 \mathrm{~cm}^{-1}$. In our experiment we observe a characteristic Davydov splitting of mode in $A_{1 g}$ in three and more layers [10]. The cause of this effects are due to occurrence of more than one $\mathrm{MoSe}_{2}$ molecule in a unit cell. Active out of-plane $E_{2 g}^{1}$ mode appears at $289 \mathrm{~cm}^{-1}$. Moreover, at $353 \mathrm{~cm}^{-1}$ we observe an inactive in-plane vibrational mode $B_{2 g}^{1}$, which intensity is the strongest for bilayer material and reduces as the number of layers increases.

We incorporate the procedure described above for multiple samples to report analysis of 18 different flakes in total.

\section{Analysis}

The experimental RRD results and the number of monolayers determined from Raman spectra analysis is summarized in Fig. 3. With increasing thickness of the sample the change of the relative reflectance was observed. We estimated the reflectance uncertainty to $\pm 0.03 \mathrm{RRD}$. The use of the difference of relative reflectance allows us to determine the exact number of TMDC layers. This method provides also a visualization of various macroscopic defects of $2 \mathrm{D}$ materials.

Transfer matrix method was used to model RRD, taking into account light reflection and propagation in the $\mathrm{MoSe}_{2}$ flake, $75 \mathrm{~nm}$ of $\mathrm{SiO}_{2}$ and $\mathrm{Si}$ substrate. Complex refractive indices at $543 \mathrm{~nm}$ and $650 \mathrm{~nm}$ were found in the literature for bulk $(4.88+1.9 \mathrm{i}$ and $4.75+1.18 \mathrm{i}$, respectively for the two laser lines, which is marked with blue solid curve in Fig. 3), $\mathrm{MoSe}_{2}$ monolayer $(5.0+1.72 \mathrm{i}$ and $4.0+1.0 \mathrm{i}$, red solid curve in Fig. 3) [1] and $\mathrm{Si} / \mathrm{SiO}_{2}$ [11]. These values were used for calculations [1]. The main 


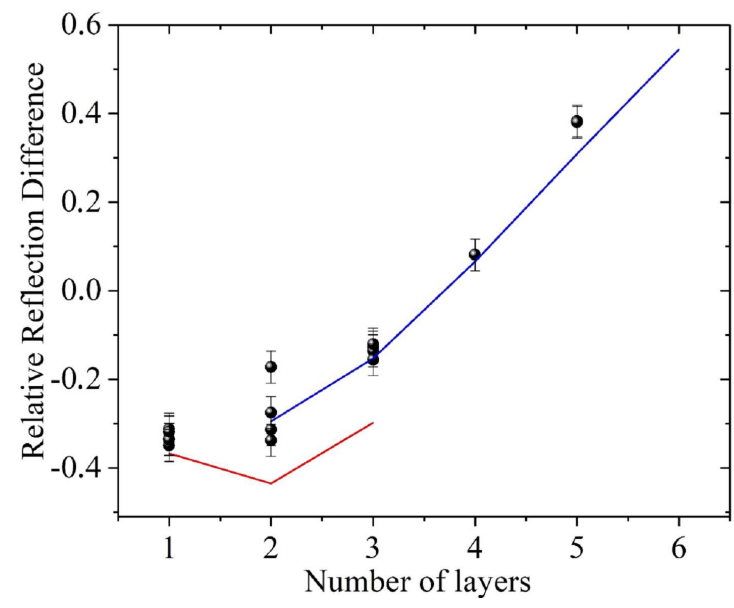

Fig. 3. The dependence of RRD (determined by confocal microscopy studies) on the number of layers (determined by Raman spectroscopy) - experimental results and theoretical modeling of TMDCs.

parameters of the transfer matrix model were the thicknesses of $\mathrm{MoSe}_{2}(1 \mathrm{~L}=0.65 \mathrm{~nm})[12]$ and its indices of refraction at a given wavelength. Resulting values of RRD as a function of layer thickness are marked by solid lines to guide the eyes. Only the integer numbers of layers have physical meaning.

Using transfer matrix modelling we could also estimate the thickness of top $\mathrm{SiO}_{2}$ substrate layer which for two given wavelengths allows for better resolution of one and two $\mathrm{MoSe}_{2}$ monolayers. The use of the silica substrate covered by $90 \mathrm{~nm} \mathrm{SiO}_{2}$ would enhance the precision of thickness determination.

\section{Conclusions}

We demonstrated that the RRD approach can be used for determination of $\mathrm{MoSe}_{2}$ flakes thicknesses. The use of two distinct laser sources allows for better contrast and thus improves the precision of the analysis comparing to previous methods based on single monochromatic light reflectivity [8]. This RRD method allows to discriminate flakes with the thickness over 2 monolayers. Between one and two $\mathrm{MoSe}_{2}$ monolayers value of RRD is unambiguous. For this case RRD method can be improved by proper choice of the $\mathrm{SiO}_{2}$ layer thickness.
Our method can be easily adapted for large area of surfaces with $\mathrm{MoSe}_{2}$ flakes, which allows for fast determination of the quality of exfoliated layers. Automatic RRD analysis of reflectivity images taken for two distinct wavelengths with standard optical microscopy will facilitate the procedure of finding a flake of a given thickness.

\section{Acknowledgments}

Scientific work was co-financed from the Ministry of Higher Education budget for education as a research project "Diamentowy Grant": 0109/DIA/2015/44 in years 2015-2019 and 0005/DIA/2016/45 in years 20162020. This work also was supported by the National Science Centre grant UMO-2013/10/M/ST3/00791.

\section{References}

[1] Y. Li, A. Chernikov, X. Zhang, A. Rigosi, H.M. Hill, A.M. van der Zande, D.A. Chenet, E.M. Shih, J. Hone, T.F. Heinz, Phys. Rev. B 90, 205422 (2014).

[2] A. Pospischil, T. Mueller, Appl. Sci. 6, 78 (2016).

[3] W. Zhao, Z. Ghorannevis, K.K. Amara, J.R. Pang, M. Toh, X. Zhang, C. Kloc, P.H. Tan, G. Eda, Nanoscale 5, 9677 (2013).

[4] Q.H. Wang, J. Coleman, M.S. Strano, Nat. Nanotechnol. 7, 699 (2012).

[5] M. Grzeszczyk, K. Gołasa, M. Zinkiewicz, K. Nogajewski, M.R. Molas, M. Potemski, A. Wysmołek, A. Babiński, 2D Materials 3, 025010 (2016).

[6] A. Castellanos-Gomez, M. Buscema, R. Molenaar, V. Singh, L. Janssen, H.S.J. van der Zant, G.A. Steele, 2D Materials 1, 011002 (2014).

[7] K.P. Dhakal, D.L. Duong, J. Lee, J. Kim, Nanoscale 6, 13028 (2014).

[8] M. Grzeszczyk, K. Gołasa, B. Piętka, A. Babiński, J. Szczytko, Acta Phys. Pol. A 126, 1207 (2014).

[9] P. Tonndorf, R. Schmidt, P. BĂśttger, X. Zhang, J. Börner, A. Liebig, M. Albrecht, C. Kloc, O. Gordan, D.R.T. Zahn, S.M. de Vasconcellos, R. Bratschitsch, Opt. Express 21, 4916 (2013).

[10] T. Sekine, M. Izumi, T. Nakashizu, K. Uchinokura, E. Matsuura, J. Phys. Soc. Jpn. 49, 1069 (1980).

[11] D.E. Aspnes, A.A. Studna, Phys. Rev. B 27, 985 (1983).

[12] A. Arora, K. Nogajewski, M. Molas, M. Koperski, M. Potemski, Nanoscale 7, 20769 (2015). 